\title{
An Unbounded Nondeterministic Model for CSP-like Languages
}

\author{
Luming Lai \\ Department of Computing, University of Bradford \\ Bradford, England
}

\begin{abstract}
The main contribution of this paper is the introduction of unbounded nondeterminism into CSP-like languages with specification, which increases the expressive power of the specification language. This is achieved by extending the finite message set of such mixed languages to an infinite one. A denotational semantics and a refinement order are defined for such a language and the CSP constructors are proved to be monotone on the specification space and continuous on the process space, which are needed for a simple treatment of iterations on both spaces of the mixed CSP-like language.
\end{abstract}

\section{Introduction}

There already exist a number of models for CSP-like languages, e.g., [6, 7, 10, 11, 12]. To exclude unbounded nondeterminism (or discontinuity of language constructors), the communication alphabets, i.e., the set of channels and messages, are restricted to be finite sets. This restriction becomes a nuisance especially when the language is extended to include specification. For example, with an infinite message set, the Fermat Theorem can be specified as follows (we know now such a specification is infeasible): (which can also be specified in CSP using an infinitary parallel composition constructor [5].)

$$
\begin{aligned}
\operatorname{var} n, a, b, c: & \mathbb{N} \bullet \\
n, a, b, c: & :\left[\text { true, } a^{n}+b^{n}=c^{n} \wedge 2<n \wedge 0<a, b, c\right] .
\end{aligned}
$$

Research has been done in recent years to handle unbounded nondeterminism in the CSP theory, e.g., [5, 11]. Unbounded nondeterminism refers to the ability of a process to choose locally one from an infinite set of possible behaviours. The difficulties encountered with unbounded nondeterminism in CSP is the loss of the fixed-point theory for defining recursions. Roscoe first tackled this problem in [11] by introducing a complete but coarser partial order on the failures-divergence model in [3]. The resulting model can successfully model a process which will, on its first step, nondeterministically choose any integer, but cannot tell between a process which can communicate any finite number of $a$ 's and one which may also choose to communicate an infinite number. To overcome this problem, the failures-divergence model is extended to include infinite traces so that any CSP process is represented by $\langle F, D, I\rangle$ where $F$ is its failures (still with finite traces), $D$ is its set of (finite) divergence traces and $I$ is the set of infinite traces it can communicate. The refined mode can model unbounded nondeterminism properly but with the loss of completeness and monotonicity. To cure this, advanced mathematics was used resulting in more difficult proofs and clumsy mathematical treatment.

In [5] Kumar and Pandya extended CSP to include an infinitary parallel composition operator to increase the expressive power of CSP. The well-formedness of the operator in the failures-divergence model was established under the syntactic restriction that an event can occur only in the alphabet of finitely many processes. Therefore, unbounded nondeterminism is excluded without the sacrifice of the elegance of the CSP theory.

In CSP's failures-divergence model, processes can have infinite alphabets without violating the continuity of CSP constructors if no infinitary nondeterministic choice operator is used and the hiding operator does not hide an infinite 
set of communication events. In this paper we follow the same route to extend the finite message set of a CSP-like language to an infinite one to increase the expressive power of the language extended with specifications. We do not, however, want to introduce unbounded nondeterminism into the CSP-like language itself because it cannot be implemented and also causes discontinuity of the program constructors and thus may need some advanced but, in practice, cumbersome techniques such as transfinite induction in [2,9]. To avoid unbounded nondeterminism, some syntactic restriction is placed on the hiding operator which can only be used to hide internal channels of a concurrent process, which is the case for most CSP-like languages. Otherwise, unbounded nondeterminism is unavoidable. For example, with an infinite message set, the following program is equivalent to choosing any value for $x$. This, therefore, introduces unbounded nondeterminism into the language, resulting the discontinuity of some of the language constructors.

$$
(c ? x) \backslash c .
$$

By restricting the hiding operator to internal channels, we prove that unbounded nondeterminism can be avoided in the CSP-like language without specification and iterations (also recursions) can be treated in the usual way.

In this paper we follow Morgan's example [8] and treat specifications like program statements, so that there is a unified framework for modelling specifications and programs [7]. Doing so, an infinite alphabet will increase the expressive power of the specification language. However, the extended model including the specification statement contains unbounded nondeterminism, resulting in discontinuity of some of the CSP constructors. We prove that all the CSP-like language constructors are monotonic with respect to the refinement order on the extended model. Therefore, the semantics of iterations can still be defined as a fixed point over all ordinals instead of only finite ones.

This paper is organised as follows. Section 2 provides a brief introduction to the mathematics used in the paper. Section 3 defines the model for the CSP-like language. Section 4 introduces a specification statement and extends the above model to include mixed terms. In Section 5 the syntax and semantics of the CSP-like language with specification are defined. Section 6 proves the monotonicity of all the CSP-like constructors. Section 7 concludes the paper.

\section{Mathematical preliminaries}

A relation on a set $C$ is a "partial ordering" of $C$ if it is reflexive, anti-symmetric, and transitive; it is a "total ordering" if in addition each pair of elements in $C$ are comparable. We denote the structure consisting of set $C$ and a partial ordering $\sqsubseteq$ on $C$ by $(C, \sqsubseteq)$. However, when the partial ordering is understood or when the context makes clear whether we are regarding $C$ as a set or as a set with a partial ordering, we do not distinguish between $C$ and $(C$, 5$)$.

A set $B \subset C$, with $C$ and hence $B$ partially ordered by $\sqsubseteq$, has an "upper bound" $u \in C$ if $x \sqsubseteq u$ for all $x \in B$; $u$ is a "least upper bound"-"lub" for short-if in addition $u \sqsubseteq v$ for every upper bound $v$ of $B$. The lub of $B$ when it exists is denoted by $\bigsqcup B$. An element $\perp \in C$ is a "bottom" or "least element" of $C$ if $\perp \sqsubseteq x$ for all $x \in C$, and $\top \in C$ is a "top" or "greatest element" of $C$ if $x \sqsubseteq \top$ for all $x \in C$. An element $m \in C$ is a "minimal element" of $C$ if $x \sqsubseteq m \Rightarrow x=m$ for all $x \in C$.

If sets $C$ and $D$ are partially ordered by $\sqsubseteq_{C}$ and $\sqsubseteq_{D}$, respectively, then the Cartesian product $C \times D$ is partially ordered by $\sqsubseteq$, defined by

$$
<x, y>\sqsubseteq<w, z>\equiv x \sqsubseteq w \text { and } y \sqsubseteq z
$$

for all $x, w \in C$ and $y, z \in D$. Definition (1) can be generalised to $n$-fold Cartesian products in the obvious way. The product sets in this paper will always be partially ordered by (1) and we will not state this explicitly in each case.

Given sets $C$ and $D$ with $D$ partially ordered by $\sqsubseteq_{D}$, the set $C \rightarrow D$ of functions from $C$ to $D$ is partially ordered by $\sqsubseteq$, defined by

$$
f \sqsubseteq g \equiv f(x) \sqsubseteq_{D} g(x) \text { for all } x \in C .
$$

In this paper, functions will be partially ordered by (2) only and we will not state this explicitly in each case. If, in addition $C$ is partially ordered by $\sqsubseteq_{C}$, then $f: C \rightarrow D$ is said to be "monotone" if

$$
x \sqsubseteq_{C} y \Rightarrow f(x) \sqsubseteq_{D} f(y) \text { for all } x, y \in C .
$$

2nd Irish Workshop on Formal Methods, 1998 
We denote by $(C \rightarrow D)$ the set of monotone functions from $C$ to $D$.

A "complete lattice" is a partially ordered set in which each subset has a lub. It can be shown that every complete lattice has a bottom and a top. We now give some well-known properties of complete lattices, omitting proofs; the reader looking for more details should refer to literature such as [1].

Lemma 1 Any finite totally ordered set is a complete lattice.

Lemma 2 If $C$ and $D$ are complete lattices, then so is $C \times D$. Moreover, for $B \subset C \times D$,

$$
\bigsqcup B=<\bigsqcup\{x:(\exists y:<x, y>\in B)\}, \bigsqcup\{y:(\exists x:<x, y>\in B)\}>
$$

Lemma 2 can be generalised to $n$-fold Cartesian products in the obvious way.

Lemma 3 If $C$ is a partially ordered set and $D$ is a complete lattice, then $(C \rightarrow D)$ is a complete lattice. Moreover, for $B \subset(C \rightarrow D)$,

$$
(\bigsqcup B)(x)=\bigsqcup\{f(x): f \in B\} \quad \text { for all } x \in C
$$

As is conventional we will use small Greek letter to denote ordinals; $\omega$ will denote the first infinite ordinal, i.e., the set of natural numbers. For any complete lattice $C$ and $f:(C \rightarrow C)$, we define

$$
\begin{aligned}
f^{0} & =\text { the identity function on } C, \\
f^{\lambda+1} & =f \circ f^{\lambda} \quad \text { (functional composition) for successor ordinals } \lambda+1, \\
f^{\lambda} & =\bigsqcup\left\{f^{\gamma}: \gamma<\lambda\right\} \text { for limit ordinals } \lambda .
\end{aligned}
$$

Lemma 4 Given $f:(C \rightarrow C)$ for $C$ a complete lattice, and $c \in C$ satisfying $c \sqsubseteq f(c)$,

1. $\beta \leq \gamma \Rightarrow f^{\beta}(c) \sqsubseteq f^{\gamma}(c)$ for all ordinals $\beta$ and $\gamma$;

2. There exists a least ordinal $\alpha$ such that

$$
\forall \gamma(\gamma \geq \alpha): f^{\gamma}(c)=f^{\alpha}(c)
$$

In Lemma 4 (2) $\alpha$ is called the "closure ordinal" of $f$ in $C$.

As we can safely replace the closure ordinal $\alpha$ in $f^{\alpha}(c)$ with any ordinal $\geq \alpha$, we can conveniently work with one "super-closure" ordinal $\infty_{f}$ for each $f$; for $\infty_{f}$ take any ordinal containing all closure ordinals of $f$. For brevity, we will write simply $\infty$, letting context supply the implicit subscript.

Any $c$ satisfying $f(c)=c$ is called a "fixed point" of $f$; if in addition $c \sqsubseteq d$ for every fixed point $d$ of $f$, then $c$ is a "least fixed point" of $f$. The least fixed point of $f$ when it exists, is denoted by $\mu x . f(x)$.

Lemma 5 Given $f:(C \rightarrow C)$ for $C$ a complete lattice with bottom $\perp, f$ has a least fixed point satisfying $\mu x . f(x)=$ $f^{\infty}(\perp)$.

Monotonicity also applies to sequences in the obvious way: a (possibly transfinite) sequence $\left\langle x_{0}, x_{1}, \cdots, x_{\alpha}, \cdots\right\rangle$ with elements drawn from a set partially ordered by $\sqsubseteq$ is said to be "monotone" if $\alpha \leq \beta \Rightarrow x_{\alpha} \sqsubseteq x_{\beta}$ for all $\alpha$ and $\beta$.

In the rest of this paper we will employ only $\sqsubseteq$ to denote a partial ordering, letting context resolve any ambiguity that might otherwise arise. 


\section{The model}

There already are some models for CSP-like languages, e.g., [6, 7, 10, 11, 12]. In this paper we take the notations in [7] (the reader may also refer to the textbook by Hoare [4]) and model a process as a set of quadruples

$$
\left(s_{0}, t r, r e f, s\right),
$$

where the first and last components $s_{0}, s(\in$ State) are machine states, which map a variable in Var (may be infinite) to a value in $\mathrm{Val}$ (may be infinite), i.e., $s_{0}, s: \mathrm{Var} \rightarrow \mathrm{Val} ; \mathrm{tr}$ is a communication trace in $\mathrm{Comm}^{*}$, which are finite sequences of communication events of the form c.v $(\in \mathrm{Comm} \hat{=}$ Chan $\times \mathrm{Val})$, including the empty one $<>$; the third component $r e f$ is a set of channel names from Chan, called refusals. Therefore, the terminating process skip that changes nothing but terminates successfully, can be defined as follows:

$$
\operatorname{skip} \hat{=}\left\{\left(s_{0},<>\text {, ref, } s_{0}\right) \mid s_{0} \in \text { State } \wedge \text { ref } \subseteq \text { Chan }\right\} \text {. }
$$

Notice that $r e f \subseteq$ Chan in (3) can be omitted because it is always true.

To model divergences, we introduce a special state $\perp$. Thus,

$$
\left(s_{0}, t r, r e f, \perp\right)
$$

represents a divergent computation after $t r$. To model non-termination, we introduce another special state $T$. Thus,

$$
\left(s_{0}, t r, r e f, \top\right)
$$

represents an unfinished computation where the final state is unobservable. The non-terminating and broken process stop can be defined as follows:

$$
\text { stop } \hat{=}\left\{\left(s_{0},<>\text {,ref }, \top\right) \mid s_{0} \in \text { State }\right\} \text {. }
$$

The enlarged state set is denoted by State ${ }_{\perp}^{\top}$.

The processes are sets of computations $\left(\subseteq C\right.$ Comp $\hat{=}$ State $\times \operatorname{Comm}^{*} \times$ Chan $\times$ State $\left._{\perp}^{\top}\right)$, which satisfy the following conditions.

Definition 1 Processes For a given communication channels Chan, a variable set Var, and a value set Val, the process space Proc is a set of all subsets $P$ of $C o m p$, which satisfy the following conditions: For any $s_{0} \in$ State,

$\mathbf{P 1} \operatorname{traces}\left(P, s_{0}\right)\left(=\left\{\operatorname{tr} \mid \exists s .\left(s_{0}, t r, r e f, s\right) \in P\right\}\right)$ is nonempty and prefix-closed:

$$
t r \neq<>\wedge\left(s_{0}, t r^{\wedge} t r, r e f, s\right) \in P \Longrightarrow\left(s_{0}, t r^{\prime},\{\}, \top\right) \in P
$$

$\mathbf{P 2}\left(s_{0}, t r, r e f, s\right) \in P \wedge r e f^{\prime} \subseteq r e f \Longrightarrow\left(s_{0}, t r, r e f^{\prime}, s\right) \in P$;

P3 $\left(s_{0}, t r, r e f, s\right) \in P \wedge \neg \exists v, s^{\prime} .\left(s_{0}, t r^{\wedge}<c . v>,\{\}, s^{\prime}\right) \in P \Longrightarrow$

$\left(s_{0}, t r, r e f \cup\{c\}, s\right) \in P$;

P4 $\left(s_{0}, t r^{\wedge}\left\langle c_{i n} . v>\right.\right.$, ref, $\left.s\right) \in P \Longrightarrow \forall v^{\prime} \exists s^{\prime} .\left(s_{0}, t r^{\wedge}\left\langle c_{i n} . v^{\prime}\right\rangle\right.$, ref, $\left.s^{\prime}\right) \in P$;

P5 $\left(s_{0}, t r, r e f, s\right) \in P \wedge s \in$ State $\Longrightarrow \forall r e f^{\prime} .\left(s_{0}, t r, r e f^{\prime}, s\right) \in P$;

P6 $\left(s_{0}, t r, r e f, s\right) \in P \wedge\left(s_{0}, t r,\{\}, \perp\right) \notin P \Longrightarrow\left\{s^{\prime} \mid\left(s_{0}, t r,\{\}, s^{\prime}\right) \in P\right\}$ is finite;

P7 $\left(s_{0}, t r, r e f, \perp\right) \in P \Longrightarrow \forall t r^{\prime}, r e f^{\prime}, s .\left(s_{0}, t r^{\wedge} t r^{\prime}, r e f^{\prime}, s\right) \in P$,

where $v$ and $v^{\prime}$ range over $\mathrm{Val}, t r$ and $t r^{\prime}$ over $C o m m^{*}$, ref and $r e f^{\prime}$ over $\mathcal{P}($ Chan $), s$ and $s^{\prime}$ over $S t a t e_{\perp}^{\top}$, and $c_{i n}$ is an input channel (not a linked channel).

$\square$ Property $\mathbf{P 4}$ states that selective input is not allowed. This property is not needed in the CSP theory because CSP deals with individual communication events. But it is essential for CSP-like languages where refusals are sets of channels. Property P5 states that if a process has a proper internal state after the current trace, it can terminate successfully in that state without doing any further communications. Property P6 states that if a process does not diverge after a trace $t r$, it can have only finitely many internal states. This excludes unbounded nondeterminism in processes and also makes sequential composition continuous on the process space Proc. 


\section{Specification statement}

A specification of a communicating process with state describes not only its communication behaviours, but also the relationships between communications, initial states and final states.

In this paper we regard a communicating processes with state as a generalised-state $(t r, s)$ transformer as in [12], which takes a process from an initial state $\left(t r_{0}, s_{0}\right)$ to a final state $\left(t r_{0}^{\wedge} t r, s\right)$ after having performed the communications in $t r$.

The syntax of the specification statement $S p$ is defined as follows:

$$
\text { Sp } \quad:: \quad I, w:[\text { pre, post }],
$$

where $w$ is a list of alterable program variables, $I$, pre, and post are predicates of the types:

$$
\begin{aligned}
& \text { pre } \left.: \text { Comm }^{*} \times \text { State } \rightarrow \text { true, false, error }\right\} \\
& \text { post : } \left.\mathrm{Comm}^{*} \times \mathrm{Comm}^{*} \times \text { State } \times \text { State } \rightarrow \text { true, false, error }\right\} \\
& I: \mathrm{Comm}^{*} \times \mathrm{Comm}^{*} \times \mathcal{P}\left(\text { Chan }_{\sqrt{ }}\right) \rightarrow\{\text { true, false, error }\} \text {, }
\end{aligned}
$$

where " $\sqrt{ }$ " represents successful termination and $A_{a}=A \cup\{a\}$.

The intuitive meaning of the specification statement is as follows. $S p$, when started in one of the initial generalisedstates satisfying $p r e\left(t r_{0}, s_{0}\right)$, must be able to engage in any communications $t r$ satisfying $I\left(t r_{0}, t r_{0}^{\wedge} t r, r e f\right)$; if $S p$ terminates, it does so in one of the final states satisfying post $\left(t_{0}, t r_{0}^{\wedge} t r, s_{0}, s\right)$, with the terminating trace $t r$ satisfying $I\left(t r_{0}, t r_{0}^{\wedge} t r,\{\}\right)$; if $S p$ cannot start in an initial state $s_{0}$, i.e., $\forall t r_{0} . \neg p r e\left(t r_{0}, s_{0}\right)$, it diverges immediately.

A special symbol " $\checkmark$ " is added to the domain of the refusals of specification statements, which indicates termination. If $\{\sqrt{ }\}$ is a possible refusal of a specification statement after $t r$, it may refuse to terminate successfully. The precise meaning of " $\sqrt{ }$ " will be given when the semantics of the specification statement is defined.

Specifications can also be defined as a set of computations in Comp. The set of all mixed terms, which are a mixture of specifications and programs, is defined as $\operatorname{Mix} T \hat{=} \mathcal{P}(\operatorname{Comp})$, which also contains all the processes defined in Def. 1. Of course, not every mixed term possesses all the properties $\mathbf{P 1}$ to $\mathbf{P 7}$ and the empty set is an example.

The refinement ordering $\sqsubseteq$ on MixT is defined the same as that on Proc:

$$
M_{1} \sqsubseteq M_{2}, \quad \text { if and only if } M_{2} \subseteq M_{1},
$$

so that the least element on MixT is also the same as that on Proc, i.e., $\perp$.

Theorem $1(M i x T, \sqsubseteq, \perp)$ and $($ Proc $, \sqsubseteq, \perp)$ are complete partial orders.

Proof. See Appendix.

\section{The CSP-like language and its semantics}

To demonstrate the effect of introducing unbounded non-determinism into a CSP-like language, we choose the following simple language.

Definition 2 The syntax of mixed terms The CSP-like language with specification statements, MProg, is defined as follows:

$$
\begin{aligned}
M::= & I, w:[\text { pre, post }] \mid \text { skip | stop | div }|x:=e| c ? x|c ! e| \\
& M_{1} ; M_{2} \mid \text { if } b \text { then } M_{1} \text { else } M_{2} \text { fi }\left|\left[M_{1}|| M_{2}\right]\right| \text { while } b \text { do } M \text { od, }
\end{aligned}
$$

where the parallel composition construct $\left[M_{1} \| M_{2}\right]$ is the same as the usual CSP construct except that the linked channels between $M_{1}$ and $M_{2}$ are hidden.

2nd Irish Workshop on Formal Methods, 1998 
We use $\llbracket \bullet \rrbracket$ to denote the main semantic function:

$$
\llbracket \bullet \rrbracket: M P r o g \rightarrow M i x T,
$$

which maps a mixed terms to a set of computations.

We first define the semantics of the specification statement.

$$
\begin{aligned}
& \llbracket I, w:[p r e, p o s t] \rrbracket \\
& \hat{=} \quad\left\{\left(s_{0}, t r, r e f, s\right) \mid \forall t r_{0} \cdot \operatorname{pre}\left(t r_{0}, s_{0}\right) \Longrightarrow I\left(t r_{0}, t r_{0}^{\wedge} t r,\{\}\right) \wedge s_{0}={ }_{w} s \wedge\right. \\
& \left.\operatorname{post}\left(t r_{0}, t r_{0}^{\wedge} t r, s_{0}, s\right)\right\} \\
& \cup\left\{\left(s_{0}, t r, r e f, \top\right) \mid \forall t r_{0} . p r e\left(t r_{0}, s_{0}\right) \Longrightarrow I\left(t r_{0}, t r_{0}^{\wedge} t r, r e f \uplus\{\sqrt{ }\}\right\}\right. \\
& \text { provided that pre, post, and } I \text { do not evaluate to error }
\end{aligned}
$$

where $\left(s_{1}={ }_{w} s_{2}\right) \hat{=} \forall x \notin w . s_{1}(x)=s_{2}(x)$ and $A \uplus\{a\} \hat{=} A \cup\{a\}$, where $a \notin A$.

Equation (5) defines the terminating computations of $S p$, as well as its divergent computations. The specification statement is a total-correctness formula in the sense that, if $S p$ has some terminating computation $\left(s_{0}, t r, r e f, s\right)$, there must exist some initial trace $t r_{0}$ such that $\operatorname{pre}\left(t_{0}, s_{0}\right)$ holds for $s_{0}$ and its terminating trace $t r$ and final state $s$, together with $t r_{0}$, satisfies the communication invariant $I\left(t r_{0}, t r_{0}^{\wedge} t r,\{\}\right)$ and the postcondition post $\left(t r_{0}, t r_{0}^{\wedge} t r, s_{0}, s\right)$. The state transition from $s_{0}$ to $s$ can only be achieved by changing those alterable variables in $w$. Whenever there exists some proper final state, termination can happen; we have, therefore, no constraints on ref in (5). If there is no initial trace satisfying $p r e\left(t r_{0}, s_{0}\right)$ for an initial state $s_{0}, S p$ diverges. In this case, $(5)=\perp$.

Equation (6) defines the non-terminating computations of $S p$. To do so, a new element " $\sqrt{ }$ " is introduced into the domain of refusals in the communication invariant $I$. Without it, unwanted nonterminating computation $\left(s_{0}, t r, r e f, \top\right)$ would also be included in $\llbracket S p \rrbracket$ for every terminating computation $\left(s_{0}, t r, r e f, s\right)$ in $\llbracket S p \rrbracket$. If $S p$ diverges in some $s_{0}$, then $(5)=(6)$.

The semantics of the rest of the language constructs are defined as follows. For expressions $e$ (including Boolean expressions), the value of $e$, evaluated in a state $s_{0}$, is denoted by $e\left(s_{0}\right)$. If anything goes wrong, such as a variable being undefined, the value of $e\left(s_{0}\right)$ is error. Whenever this happens, $\llbracket \bullet \rrbracket$ produces a divergent process.

The divergent process div is an unspecified process on whose behaviour there is no constraint whatsoever. It is identified with the least element $\perp$ on $\operatorname{Mix} T$ :

$$
\llbracket \operatorname{div} \rrbracket \hat{=} \perp \text {. }
$$

The definitions for skip and stop are already given in (3) and (4) in Section 3, respectively. The assignment statement, $x:=e$, can be defined as follows:

$$
\llbracket x:=e \rrbracket \hat{=}\left\{\left(s_{0},<>, \text { ref, } s_{0}\left[e\left(s_{0}\right) / x\right]\right) \mid s_{0} \in \text { State }\right\}, \quad \text { where } e\left(s_{0}\right) \neq \text { error }
$$

and $s[v / x]$ is the same as $s$ except that the value of $x$ is $v$.

The input process $c$ ? $x$ inputs a message on channel $c$ and stores it in $x$.

$$
\begin{aligned}
&\llbracket c ? x] \widehat{\hat{=}}\left\{\left(s_{0},<>, \text { ref }, \top\right) \mid s_{0} \in \text { State } \wedge c \notin \text { ref }\right\} \\
& \cup\left\{\left(s_{0},<c . v>, \text { ref }, s_{0}[v / x]\right) \mid s_{0} \in \text { State } \wedge v \in \text { Val }\right\} .
\end{aligned}
$$

Notice that the second set above is infinite as Val is infinite.

The semantics of the output process $c ! e$ is similar to that of $c ? x$ and left with interested readers.

If $M_{1}$ and $M_{2}$ are two mixed terms with the same alphabets, then $\left(M_{1} ; M_{2}\right)$ is a mixed term which behaves like $M_{1}$, except that if $M_{1}$ terminates successfully, it continues behaving like $M_{2}$. Its definition is

$$
\begin{aligned}
\hat{=} \quad & \llbracket M_{1} ; M_{2} \rrbracket \\
& \left\{\left(s_{0}, t r, r e f, \top\right) \mid\left(s_{0}, t r, r e f, \top\right) \in \llbracket M_{1} \rrbracket\right\} \\
& \cup\left\{\left(s_{0}, t r^{\wedge} t r^{\prime}, r e f^{\prime}, s\right) \mid\left(s_{0}, t r, r e f, \perp\right) \in \llbracket M_{1} \rrbracket\right\} \\
& \cup\left\{\left(s_{0}, t r^{\wedge} t r^{\prime}, r e f, s\right) \mid \exists s^{\prime} .\left(s_{0}, t r,\{\}, s^{\prime}\right) \in \llbracket M_{1} \rrbracket \wedge\left(s^{\prime}, t r^{\prime}, r e f, s\right) \in \llbracket M_{2} \rrbracket\right\}
\end{aligned}
$$


if $b$ then $M_{1}$ else $M_{2} \mathrm{fi}$ is the usual if-statement in the sequential language. If states are hidden, it behaves like the nondeterministic choice in CSP. Its definition is

$$
\begin{aligned}
& \text { 【if } b \text { then } M_{1} \text { else } M_{2} \text { fi } \\
& \hat{=} \quad\left\{\left(s_{0}, t r, r e f, s\right) \mid b\left(s_{0}\right) \wedge\left(s_{0}, t r, r e f, s\right) \in \llbracket M_{1} \rrbracket\right\} \\
& \cup\left\{\left(s_{0}, t r, r e f, s\right) \mid \neg b\left(s_{0}\right) \wedge\left(s_{0}, t r, r e f, s\right) \in \llbracket M_{2} \rrbracket\right\} . \\
& \text { provided that } b\left(s_{0}\right) \neq \text { error }
\end{aligned}
$$

The parallel composition constructor $\left[M_{1} \| M_{2}\right]$ is the key constructor in any parallel language. It is used to construct concurrent systems from individual ones. We stipulate that $M_{1}$ and $M_{2}$ do not share any program variable other than read-only ones. The communication alphabet of $\left[M_{1} \| M_{2}\right]$ is the union of the communication alphabets of $M_{1}$ and $M_{2}$. The common channels of $M_{1}$ and $M_{2}$ are linked channels on which $M_{1}$ and $M_{2}$ can communicate. Linked channels are hidden from outside. Therefore, the parallel constructor is a combination of the CSP parallel constructor $\|$ and hiding operator $\backslash$. We define it as follows:

$$
\left[M_{1} \| M_{2}\right] \hat{=}\left(M_{1} \| M_{2}\right) \backslash\{\text { linked channels }\}
$$

where the parallel composition $\|$ and the hiding operator are defined as follow.

$$
\begin{gathered}
\hat{=} \quad\left\{\left(s_{0}, t r, r e f_{1} \cup r e f_{2}, s_{1} \oplus s_{2}\right) \mid \bigwedge_{i=1,2}\left(s_{0}, t r \mid \alpha M_{i}, r e f_{i}, s_{i}\right) \in \llbracket M_{i} \rrbracket\right\} \\
\bigcup_{i, j \in\{1,2\} \wedge i \neq j}\left\{\left(s_{0}, t r, r e f, s\right) \mid \exists \operatorname{tr}^{\prime} \leq \operatorname{tr} .\left(s_{0}, \operatorname{tr}^{\prime} \mid \alpha M_{i},\{\}, \perp\right) \in \llbracket M_{i} \rrbracket \wedge\right. \\
\left.\operatorname{tr}^{\prime} \mid \alpha M_{j} \in \operatorname{traces}\left(M_{j}, s_{0}\right)\right\},
\end{gathered}
$$

where $\operatorname{tr}\lceil A$ is a trace obtained from $\operatorname{tr}$ by removing all the events that happened on channels not in $A$ and $\oplus$ is defined as follows:

$$
\left(s_{1} \oplus s_{2}\right)(x)= \begin{cases}\top, & \text { if either } s_{1}=\top \text { or } s_{2}=\top \\ s_{1}(x), & \text { otherwise, if } s_{1} \neq \perp \\ s_{2}(x), & \text { otherwise, if } s_{2} \neq \perp \\ \perp, & \text { otherwise }\end{cases}
$$

If $s_{1}$ and $s_{2}$ map $x$ into different non- $\perp$ values, the parallel combination is broken. The disjointness constraint of $\|$ guarantees that this won't happen.

The hiding operator $\backslash c$ is defined as follows. Let $M$ be a process with $c \in \alpha M$. The communication alphabet of $M \backslash c$ is that of $M$ minus $c$.

$$
\begin{aligned}
& \llbracket M \backslash c \rrbracket \\
& \hat{=} \quad\left\{\left(s_{0}, t r \backslash c, r e f, s\right) \mid c \notin r e f \wedge\left(s_{0}, t r, r e f \cup\{c\}, s\right) \in \llbracket M \rrbracket\right\} \\
& \cup\left\{\left(s_{0}, t r^{\wedge} t r^{\prime}, r e f, s\right) \mid\left\{t r^{\prime \prime} \mid t r^{\prime \prime} \backslash c=t r \wedge\right.\right. \\
& \left.\left.t r^{\prime \prime} \in \operatorname{traces}\left(M, s_{0}\right)\right\} \text { is infinite }\right\} \text {, }
\end{aligned}
$$

where $\operatorname{tr} \backslash c=\operatorname{tr}\lceil(\alpha M-\{c\})$.

The first clause claims that if $M$ is ready to communicate with its environment along some channels other than $c$, then so can $M \backslash c$; the second clause states that if $M$ engages in an infinite unbroken sequence of communications along channel $c$, then $M \backslash c$ diverges. This also includes the case where $M$ diverges.

The while statement has the usual meaning as that in sequential languages except that it also involves communications and may not terminate. It is defined as a fixed-point of the following equation:

$$
X=\text { if } b \text { then }(M ; X) \text { else skip. }
$$


However, as our extended model contains unbounded non-determinism, some of the language constructors are no longer continuous. In [2], Boom showed how Dijkstra's definition of the weakest precondition for the while loop can be adapted to permit unbounded nondeterminism. We use the same idea to adapt the definition for recursion so that unbounded nondeterminism is permitted. The basic idea is to take the infinite join over the set of all ordinals, rather than just over the set of finite ordinals. The existence of the fixed-point then depends on the monotonicity of all the language constructors which have been proved in Theorem 3.

We defined the fixed-point over ordinals by Lemma 5 :

$$
\llbracket \text { while } b \text { do } M \text { end } \rrbracket \hat{=} \bigsqcup_{\alpha \text { is an ordinal }} \llbracket H^{\alpha}(\operatorname{div}) \rrbracket,
$$

where

$$
\begin{aligned}
H^{0}(X) & \hat{=} X \\
H^{\lambda+1}(X) & \hat{=} \text { if } b \text { then }\left(M ; H^{\lambda}(X)\right) \text { else skip } \mathbf{f i} \\
H^{\lambda}(X) & \hat{=} \bigsqcup_{\alpha<\lambda} H^{\alpha}(X), \quad \text { where } \lambda \text { is a limit ordinal. }
\end{aligned}
$$

However, the CSP-like language without specification statement is continuous with respect to the refinement order on the process space Proc, as indicated in Theo. 2. Therefore, the fixed-point of the the above recursive definition does exist over the set of all finite ordinals and the traditional loop definition works.

Theorem 2 All the processes without specification are well-defined and continuous.

Proof. Since the only differences between our CSP-like language and others, such as those in [6, 12], are the infinite message set and the hiding operator which hides only internal channels, the proofs of the well-definedness and continuity of the language constructors are the same as those in [6] except for the hiding operator. We only give details for the proof of the well-definedness of the hiding operator $\backslash$. The proof of continuity of hiding follows a similar argument and is omitted here.

We prove that $P \backslash c$, where $c$ is a linked channel, satisfies conditions P1 to P7. We only give details for $\mathbf{P 6}$ which is affected by an infinite message set.

Assume $\left(s_{0}, t r, r e f, s\right) \in \llbracket P \backslash c \rrbracket$ and $\left(s_{0}, t r,\{\}, \perp\right) \notin \llbracket P \backslash c \rrbracket$. By definition, there exist some traces $t r^{\prime}$ such that $t r=t r^{\prime} \backslash c$ and $\left(s_{0}, t r^{\prime}, r e f \cup\{c\}, s\right) \in \llbracket P \rrbracket$. $P$, being a process, has property P6. Hence, $\left.\left\{s^{\prime} \mid\left(s_{0}, t r^{\prime},\{\}, s^{\prime}\right) \in \llbracket P\right]\right\}$ is finite for every $t r^{\prime}$. As $t r$ is finite, it has the form

$$
\operatorname{tr}=a_{1} a_{2} \cdots a_{n}
$$

where $a_{i}$ is a communication event on a channel other that $c$. Therefore, each trace $t r^{\prime}$ must have the form

$$
t r^{\prime}=t r_{c}^{1} a_{1} t r_{c}^{2} a_{2} \cdots a_{n} t r_{c}^{n+1},
$$

where $t r_{c}^{i}$ is a finite trace from $\{\langle c . v\rangle \mid v \in V a l\}^{*}$. As $t r^{\prime}$ is not a diverging trace of $P$ by definition and $c$ is not an input channel (see P4), there exist only finitely many $t r_{c}^{i}$, s such that $t r=t r^{\prime} \backslash c$. Hence, $\cup_{t r^{\prime}}: t r=t r^{\prime} \backslash c\left\{s^{\prime} \mid\right.$ $\left.\left.\left(s_{0}, t r^{\prime},\{\}, s^{\prime}\right) \in \llbracket P\right]\right]$ is finite; so is $\left.\left\{s^{\prime} \mid\left(s_{0}, t r,\{\}, s^{\prime}\right) \in \llbracket P \backslash c\right] \rrbracket\right\}$ as required.

\section{Monotonicity}

In this section we show that all the statement constructors, sequential composition, conditional composition, iteration, and parallel composition, are monotone with respect to the refinement order.

Sequential composition. Let $P_{i}$ and $Q_{i}$ be mixed terms satisfying $P_{i} \sqsubseteq Q_{i}$ for $i=1,2$. We prove that $P_{1} ; P_{2} \sqsubseteq$ $Q_{1} ; Q_{2}$, that is, $\llbracket Q_{1} ; Q_{2} \rrbracket \subseteq \llbracket P_{1} ; P_{2} \rrbracket$. 
Assume $\left(s_{0}, t r, r e f, s\right) \in \llbracket Q_{1} ; Q_{2} \rrbracket$. By definition of sequential composition, there are two cases. First, $\left(s_{0}, t r, r e f, s\right)$ is a non-terminating computation of $Q_{1}$, that is $\left(s_{0}, t r, r e f, s\right) \in(7) \cup(8)$ in the definition of the sequential composition. By assumption and definition of $\sqsubseteq$, we have $\left(s_{0}, t r, r e f, s\right) \in \llbracket P_{1} \rrbracket$, which means $\left(s_{0}, t r, r e f, s\right) \in$ $\llbracket P_{1} ; P_{2} \rrbracket$ by definition of sequential composition.

Secondly, $\left(s_{0}, t r, r e f, s\right) \in(9)$. Then, there exist $s^{\prime} \in$ State, $t r^{\prime}$, and $t r^{\prime \prime}$, such that $t r=t r^{\prime \wedge} t^{\prime \prime},\left(s_{0}, t r^{\prime},\{\}, s^{\prime}\right) \in$ $\llbracket Q_{1} \rrbracket$, and $\left(s^{\prime}, t r^{\prime \prime}, r e f, s\right) \in \llbracket Q_{2} \rrbracket$. By assumption that $P_{i} \sqsubseteq Q_{i}$ for $i=1,2$, we have $\left(s_{0}, t r^{\prime},\{\}, s^{\prime}\right) \in \llbracket P_{1} \rrbracket$ and $\left(s^{\prime}, t r^{\prime \prime}, r e f, s\right) \in \llbracket P_{2} \rrbracket$, which means, by the definition of the sequential composition, $\left(s_{0}, t r, r e f, s\right) \in \llbracket P_{1} ; P_{2} \rrbracket$.

Conditional composition. Let the assumption be the same as in the previous case. We prove that

$$
\text { if } b \text { then } P_{1} \text { else } P_{2} \mathbf{f i} \sqsubseteq \text { if } b \text { then } Q_{1} \text { else } Q_{2} \text { fi. }
$$

Assume ( $\left.s_{0}, t r, r e f, s\right) \in \llbracket$ if $b$ then $Q_{1}$ else $Q_{2}$ fi $\rrbracket$. Then, by definition, it belongs to either $\llbracket Q_{1} \rrbracket$, if $b\left(s_{0}\right)$ evaluates to true, or $\llbracket Q_{2} \rrbracket$, otherwise. By the assumption, it will also belongs to either $\llbracket P_{1} \rrbracket$, or $\llbracket P_{2} \rrbracket$, respectively. That is, $\left(s_{0}, t r, r e f, s\right) \in \llbracket$ if $b$ then $P_{1}$ else $P_{2}$ fi $\rrbracket$ by definition.

Parallel composition. Since the parallel composition hides its linked channels, it is a composition of the parallel constructor $\|$ and the hiding operator $\backslash$. We only need to prove that both are monotone since the functional composition of two monotone functions is also monotone.

Let $P_{i}$ and $Q_{i}$ be mixed terms satisfying $P_{i} \sqsubseteq Q_{i}$, for $i=1,2$. We prove that $P_{1}\left\|P_{2} \sqsubseteq Q_{1}\right\| Q_{2}$. Assume $\left(s_{0}, t r, r e f, s\right) \in \llbracket Q_{1} \| Q_{2} \rrbracket$. By the definition of the parallel composition, there are two cases. First, $\left(s_{0}, t r, r e f, s\right)$ is a non-diverging computation, that is, $\left(s_{0}, t r, r e f, s\right) \in(12)$. By assumption and definition of $\sqsubseteq$, we have $\left(s_{0}, t r, r e f, s\right) \in \llbracket P_{1} \| P_{2} \rrbracket$. If $\left(s_{0}, t r, r e f, s\right)$ is a diverging computation, i.e., $\left(s_{0}, t r, r e f, s\right) \in(13)$, the same argument applies. Therefore, the parallel operator is monotone.

Now assume $P \sqsubseteq Q$. We prove that $P \backslash c \sqsubseteq Q \backslash c$ where $c$ is a linked channel of $P$ and $Q$. Assume $\left(s_{0}, t r, r e f, s\right) \in$ $\llbracket Q \backslash c \rrbracket$. By definition of hiding, there are two cases. First, $\left(s_{0}, t r, r e f, s\right)$ is a non-diverging computation, that is ,$\left(s_{0}, t r, r e f, s\right) \in(15)$. Then, there exists a $t r^{\prime}$ such that $t r=t r^{\prime} \backslash c$ and $\left(s_{0}, t r^{\prime}, r e f \cup\{c\}, s\right) \in \llbracket Q \rrbracket$. By assumption, we also have $\left(s_{0}, t r^{\prime}, r e f \cup\{c\}, s\right) \in \llbracket P \rrbracket$. Hence, by definition of $\backslash,\left(s_{0}, t r, r e f, s\right) \in \llbracket P \backslash c \rrbracket$ as required. The diverging case is similar and omitted.

Iteration. First, we prove that for any mixed terms $M(X)$ and $N(X), M(X) \leq N(X)$ implies $\mu X . M(X) \leq$ $\mu$. $N(X)$. As our mixed terms allow unbounded non-determinism, we prove first by transfinite induction that $M^{\alpha}(X) \sqsubseteq N^{\alpha}(X)$, for every ordinal $\alpha$.

For $\alpha=0$, the result is trivial. For $\alpha+1$, we have that

$$
M^{\alpha+1}(X)=M\left(M^{\alpha}(X)\right) .
$$

By induction hypothesis and monotonicity of $M$, we have $M\left(M^{\alpha}(X)\right) \sqsubseteq M\left(N^{\alpha}(X)\right)$, and $M\left(N^{\alpha}(X)\right) \sqsubseteq N\left(N^{\alpha}(X)\right)$, which is $N^{\alpha+1}(X)$ as required.

For a limit ordinal $\lambda$, we have

$$
H^{\lambda}(X) \hat{=} \bigsqcup_{\alpha<\lambda} H^{\alpha}(X)
$$

By induction hypothesis that $M^{\alpha}(X) \sqsubseteq N^{\alpha}(X)$ for every ordinal $\alpha<\lambda$, we have

$$
M^{\alpha}(X) \sqsubseteq \bigsqcup_{\alpha<\lambda} N^{\alpha}(X),
$$

for every $\alpha<\lambda$. This gives us

$$
\bigsqcup_{\alpha<\lambda} M^{\alpha}(X) \sqsubseteq \bigsqcup_{\alpha<\lambda} N^{\alpha}(X),
$$

that is, the result also holds for the limit ordinal $\lambda$. Thus the result holds for every ordinal $\alpha$. 
Essentially the same argument as for the limit ordinal now gives us the main result. We have $M^{\alpha}(X) \sqsubseteq N^{\alpha}(X)$ for every ordinal $\alpha$. Hence,

$$
M^{\alpha}(X) \sqsubseteq \bigsqcup_{\alpha \text { is an ordinal }} N^{\alpha}(X)
$$

for every ordinal $\alpha$. Hence,

$$
\bigsqcup_{\alpha \text { is an ordinal }} M^{\alpha}(X) \sqsubseteq \bigsqcup_{\alpha \text { an ordinal }} N^{\alpha}(X),
$$

that is,

$$
\mu \cdot M(X) \sqsubseteq \mu \cdot N(X) .
$$

The monotonicity of the while-statement follows from the monotonicity of the above constructors. It is defined as

$$
\text { while } b \text { do } M \text { od } \hat{=} \mu X \text {. if } b \text { then }(M ; X) \text { else skip. }
$$

Assume that $M \sqsubseteq M^{\prime}$. Since $X \sqsubseteq X, M ; X \sqsubseteq M^{\prime} ; X$, by monotonicity of sequential composition. We then have, by monotonicity of if-statement,

$$
\text { if } b \text { then }(M ; X) \text { else skip } \sqsubseteq \text { if } b \text { then }\left(M^{\prime} ; X\right) \text { else skip, }
$$

Hence, by monotonicity of recursion, we have

$$
\text { while } b \text { do } M \text { od } \sqsubseteq \text { while } b \text { do } M^{\prime} \text { od. }
$$

Theorem 3 Let $M(P)$ denote a mixed term containing a substatement $P$ and $Q$ be other mixed term. Replacing $P$ by $Q$ in $S$ gives $M(Q)$. Then $P \sqsubseteq Q$ implies $M(P) \sqsubseteq M(Q)$.

Proof. The proof is straightforward by induction on the structure of $M$.

\section{Conclusion}

In this paper we extend the model for CSP-like languages to include specification statements. The restriction on the finite message set is lifted which results in the inclusion of unbounded non-determinism in the extended model and increases the expressive power of the resulting mixed specification language. We prove that all the process constructors are monotone with respect to the refinement order $\sqsubseteq$ and the fixed-points exit over ordinals. We also prove that all the language constructors are well-defined and still continuous with respect to the refinement order on the process space, which means the conventional treatment of recursion is valid for all the processes which do not contain specification statements.

\section{Acknowledgment}

The author would like to thank Dr Jeff Sanders and Prof Imad Torsun for their comments and encouragement.

\section{References}

[1] G. Birkhoff. Lattice theory. American Mathematical Society, Providence, RI, 1961.

[2] H.J. Boom. A weaker precondition for loops. ACM TOPLAS 4(4), pp. 668-677, 1982.

[3] S.D. Brookes and A.W. Roscoe. An improved failures model for communicating processes. Lecture Notes in Computer Science, 197, pp. 281-305, Springer-Verlag, 1984.

2nd Irish Workshop on Formal Methods, 1998 
[4] C.A.R. Hoare. Communicating Sequential Processes. Prentice-Hall, London, 1985.

[5] K.N.Kumar and P.K. Pandya. Infinitary parallelism without unbounded nondeterminism in CSP. Acta Informatica 30, pp. 467-487, 1993.

[6] L. Lai and J.W. Sanders. A refinement calculus for communicating processes with state. In Gerard ORegan and Sharon Flynn (eds) 1st Irish Formal Methods Workshop, Electronic Workshops in Computing. Springer-Verlag. Dublin, 1997.

[7] L. Lai. A unified model for CSP-like languages with specifications. In G. von Bochmann, R. Dssouli, and O. Rafiq (eds), Formal Descriptions Techniques VIII, Chapman \& Hall, 1996.

[8] C.C. Morgan. Programming from Specifications. Prentice-Hall, London, 1990.

[9] J.M. Morris. A theoretical basis for stepwise refinement and the programming calculus. Science of Computer Programming, 9(3), pp. 287-306, 1987.

[10] A. W. Roscoe. Denotational semantics for occam. Lecture Notes in Computer Science, 197, pp. 306-329, Springer-Verlag, 1984.

[11] A. W. Roscoe. Two papers on CSP. Technical Monograph PRG-67, PRG, Computing Laboratory, Oxford University, 1988.

[12] J. Zwiers. Compositionality, concurrency and partial correctness. Lecture Notes in Computer Science, 321, Springer-Verlag, 1989.

\section{A Appendix}

Theorem $1(M i x T, \sqsubseteq, \perp)$ and $(\operatorname{Proc}, \sqsubseteq, \perp)$ are complete partial orders.

Proof. The proof of $(\operatorname{Mix} T, \sqsubseteq, \perp)$ being a complete partial order is simple and omitted. We only prove the second part of the theorem.

We must show that every chain of processes has a least upper bound, i.e., if $\left(P_{n}\right)_{n \geq 0}$ is a chain of processes, then $\sqcup_{n \geq 0} P_{n}\left(=\cap_{n \geq 0} P_{n}\right)$ is a process, which has all the properties of a process. We only give details for $\mathbf{P 3}$ and $\mathbf{P 6}$.

The proof of P3. Suppose

$$
\left(\begin{array}{c}
\left(s_{0}, t r, r e f, s\right) \in \bigsqcup_{n \geq 0} P_{n} \\
\neg \exists v, s^{\prime} .\left(s_{0}, t r^{\wedge}<c . v>,\{\}, s^{\prime}\right) \in \bigsqcup_{n \geq 0} P_{n}
\end{array}\right) .
$$

This means that, for all $n \geq 0$,

$$
\left(s_{0}, t r, r e f, s\right) \in P_{n} .
$$

To prove that $\left(s_{0}, t r, r e f \cup\{c\}, s\right) \in \sqcup_{n \geq 0} P_{n}$, we first prove that there are infinitely many $P_{n}$ such that

$$
\neg v, s^{\prime} .\left(s_{0}, t r^{\wedge}<c . v>,\{\}, s^{\prime}\right) \in P_{n} .
$$

Then $P_{n}$, being a process, has property $\mathbf{P 3}$, which gives, for infinitely many $n$,

$$
\left(s_{0}, t r, r e f \cup\{c\}, s\right) \in P_{n} .
$$

Since $\left(P_{n}\right)_{n}$ form a chain, it follows that

$$
\left(s_{0}, t r, r e f \cup\{c\}, s\right) \in \bigcap_{n \geq 0} P_{n}\left(=\bigsqcup_{n \geq 0} P_{n}\right) .
$$

2nd Irish Workshop on Formal Methods, 1998 
Suppose there were not such infinitely many $P_{n}$. Then there would be infinitely many $i, v_{i}$, and $s_{i}$ such that $\left(s_{0}, t r^{\wedge}<c . v_{i}>,\{\}, s_{i}\right) \in P_{i}$. By $\mathbf{P 4}$ and $\left(P_{n}\right)_{n}$ being a chain, there would exist a $v^{\prime}$ such that, for infinitely many $i$,

$$
\left(s_{0}, t r^{\wedge}<c . v^{\prime}>,\{\}, s_{i}\right) \in P_{i} .
$$

$P_{i}$, being a process, has property $\mathbf{P 6}$, which gives

$$
\left\{s_{i} \mid\left(s_{0}, t r^{\wedge}<c . v^{\prime}>,\{\}, s_{i}\right) \in P_{i}\right\} \text { is finite. }
$$

Note that $t r$ cannot be a divergent trace of infinitely many $P_{i}$ because this would contradict the assumption.

Since $\left(P_{n}\right)_{n}$ form a chain, there would exists an $m$ such that

$$
\begin{aligned}
& \forall n \geq m .\left\{s_{m} \mid\left(s_{0}, t r^{\wedge}<c . v^{\prime}>,\{\}, s_{m}\right) \in P_{m}\right\} \\
= & \left\{s_{n} \mid\left(s_{0}, t r^{\wedge}<c . v^{\prime}>,\{\}, s_{n}\right) \in P_{n}\right\} .
\end{aligned}
$$

Choosing an $s^{\prime}$ from such a set, we would have, for infinitely many $n$,

$$
\left(s_{0}, t r^{\wedge}<c . v^{\prime}>,\{\}, s^{\prime}\right) \in P_{n},
$$

and then

$$
\left(s_{0}, t r^{\wedge}<c . v^{\prime}>,\{\}, s^{\prime}\right) \in \bigcap_{n \geq 0} P_{n} .
$$

This would contradict our assumption that

$$
\neg \exists v, s^{\prime} .\left(s_{0}, t r^{\wedge}<c . v>,\{\}, s^{\prime}\right) \in \bigcap_{n \geq 0} P_{n} .
$$

The proof of P6. Suppose

$$
\left(\begin{array}{c}
\left(s_{0}, t r, r e f, s\right) \in \bigsqcup_{n \geq 0} P_{n} \\
\left(s_{0}, t r,\{\}, \perp\right) \notin \bigsqcup_{n \geq 0} P_{n}
\end{array}\right) .
$$

Then there must be a process, say $P_{m}$, such that

$$
\left(\begin{array}{c}
\left(s_{0}, t r, r e f, s\right) \in P_{m} \\
\left(s_{0}, t r,\{\}, \perp\right) \notin P_{m}
\end{array}\right) .
$$

$P_{m}$, being a process, has property $\mathbf{P 6}$, which gives

$$
\left\{s^{\prime} \mid\left(s_{0}, t r,\{\}, s^{\prime}\right) \in P_{m}\right\} \text { is finite. }
$$

Since $\left(P_{n}\right)_{n}$ form a chain, it follows that

$$
\forall n \geq m .\left\{s_{n} \mid\left(s_{0}, t r,\{\}, s_{n}\right) \in P_{n}\right\} \subseteq\left\{s_{m} \mid\left(s_{0}, t r,\{\}, s_{m}\right) \in P_{m}\right\} .
$$

Therefore, we have

$$
\bigcap_{n \geq 0}\left\{s^{\prime} \mid\left(s_{0}, t r,\{\}, s^{\prime}\right) \in P_{m}\right\} \text { is finite. }
$$

